\title{
Plane strain bending under tension as an ideal flow process in pressure - dependent plasticity
}

\author{
Sergei Alexandrov ${ }^{1}$ and Prashant Date ${ }^{2}$ \\ ${ }^{1}$ Russian Academy of Sciences, Institute for Problems in Mechanics, 199526 Moscow, Russia \\ ${ }^{2}$ IIT Bombay, Department of Mechanical Engineering, 400076 Mumbai, India
}

\begin{abstract}
Ideal plastic flows are those for which all material elements follow minimum work paths. Ideal flow solutions are widely used as the basis for inverse methods for the preliminary design of metalworking processes. The present paper provides the first ideal flow solution in pressure-dependent plasticity. In particular, the process of bending under tension is considered and it is shown that there are relations between the bending moment and tensile force that result in ideal flow paths.
\end{abstract}

\section{Introduction}

Ideal plastic flows are those for which all material elements follow minimum work paths. The ideal flow theory has long been associated with the Tresca yield criterion $[1,2]$. Recently, it has been demonstrated that ideal flow solutions exist in anisotropic plasticity [3]. In particular, the theory of anisotropic plasticity proposed in [4] has been adopted. A comprehensive overview of the ideal flow theory and corresonding solutions has been provided in [5]. This theory has been widely used as the basis for inverse methods for the preliminary design of bulk metal forming processes driven by minimum plastic work (see, for example, [6]). In particular, optimal dies for extrusion (or drawing) have been found in this paper. Necessary conditions for the optimality of extrusion dies for rigid plastic materials obeying strictly convex yield criteria have been derived in [7]. The development of simplified methods for analysis and design of metal forming processes is of importance for applications since standard finite element simulations are too slow for many purposes [8]. It is worthy of note that the ideal flow theory results in rather a general method of analyzing and designing metal forming processes, whereas many other simplified methods deal with a specific process (see, for example, [9] for plate rolling and [10] for wire drawing). All the available ideal fow solutions are for pressureindependent models. On the other hand, pressuredependency is an important property of some metals, for example [11 - 14]. The present paper provides the first ideal flow solution in pressure-dependent plasticity. In particular, the double shearing model proposed in [15] is adopted. The solution describes the process of bending under tension satisfying the ideal flow conditions. Non ideal flow solutions for this process have been given in [16 - 20] for various material models. The present solution is based on the mapping between Eulerian and Lagrangian coordinates proposed in [21].

\section{Statement of the problem}

The process of plane strain bending under tension transforms an initial rectangular $A_{0} B_{0} C_{0} D_{0}$ into a shape determined by two circular arcs, $A B$ and $C D$, and two straight lines, $A D$ and $B C$. The initial shape is shown in Fig. 1 and the final shape in Fig. 2. The initial thickness of the sheet is $H$ and the initial width is $L$. The sheet is loaded by bending moment $M$ and force $F$ per unit length. A consequence of the equilibrium equations is that some pressure $p$ should be applied to surface $C D$. Assuming that this pressure is uniformly distributed over the surface it is possible to find that

$$
F=r_{C D} p
$$

where $r_{C D}$ is the current radius of surface $C D$. Kinematics of this process has been described in [21]. For completeness, the basic equations are presented in this section. Choose an Eulerian Cartesian coordinate system $(x, y)$ such that the initial rectangular is defined by the equations $x=0, x=-H$ and $y= \pm L$ and a Lagrangian coordinate system $(\zeta, \eta)$ such that $x=H \zeta$ and $y=H \eta$ at the initial instant. The mapping between these coordinate systems is

$$
\begin{aligned}
& \frac{x}{H}=\sqrt{\frac{\zeta}{a}+\frac{s}{a^{2}}} \cos (2 a \eta)-\frac{\sqrt{s}}{a}, \\
& \frac{y}{H}=\sqrt{\frac{\zeta}{a}+\frac{s}{a^{2}}} \sin (2 a \eta)
\end{aligned}
$$




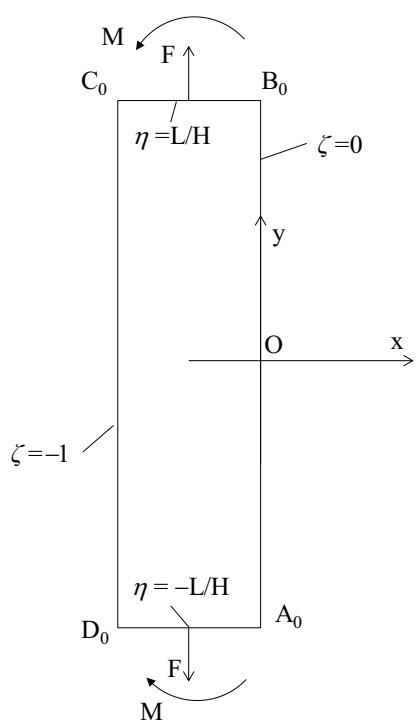

Figure 1. Initial configuration.

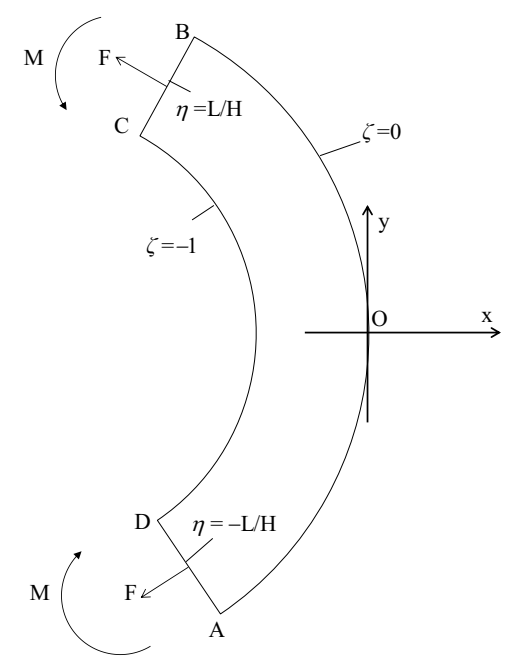

Figure 2. Final configuration.

where $a$ is a function of the time, $t$, satisfying the condition $a=0$ at $t=0$ and $s$ is a function of a satisfying the condition

$$
s=\frac{1}{4}
$$

at $a=0$. The function $s(a)$ should be found from the solution. It is possible to verify by inspection that Eq. (2) satisfies the equation of incompressibility. Moreover, the Lagrangian coordinate system is orthogonal and its coordinate curves coincide with trajectories of principal strain rates. It is also convenient to introduce a moving plane polar coordinate system $(r, \theta)$ with its origin at $x=-H \sqrt{s} / a$ and $y=0$. Then, it follows from Eq. (2) that

$$
\frac{r}{H}=\frac{\sqrt{\zeta a+s}}{a}, \quad \theta=2 a \eta .
$$

In this coordinate system, curves $A B$ and $C D$ are defined by the equations $r=r_{A B}$ and $r=r_{C D}$, respectively, and lines $C B$ and $A D$ by the equations $\theta= \pm \theta_{0}$. It follows from Eq. (4) that

$$
\frac{r_{A B}}{H}=\frac{\sqrt{s}}{a}, \quad \frac{r_{C D}}{H}=\frac{\sqrt{s-a}}{a}, \quad \theta_{0}=\frac{2 a L}{H} .
$$

Using these equations the bending moment is expressed as

$$
M=\int_{r_{C D}}^{r_{A B}}\left(\sigma_{\theta}-\frac{F}{h}\right) r d r .
$$

Here $\sigma_{\theta}$ is the circumferential stress ( $\sigma_{r}$ will stand for the radial stress) and $h=r_{A B}-r_{C D}$. The principal strain rate components, $\xi_{\zeta}$ and $\xi_{\eta}$, can be found from Eq. (2) as

$$
\begin{aligned}
& \xi_{\zeta}=\xi_{r r}=-\frac{(\zeta+\mathrm{d} s / \mathrm{d} a)}{2(\zeta a+s)} \frac{\mathrm{d} a}{\mathrm{~d} t} \\
& \xi_{\eta}=\xi_{\theta \theta}=\frac{(\zeta+\mathrm{d} s / \mathrm{d} a)}{2(\zeta a+s)} \frac{\mathrm{d} a}{\mathrm{~d} t}
\end{aligned}
$$

Here $\xi_{r r}$ and $\xi_{\theta \theta}$ are the normal strain rates in the plane polar coordinate system. In the case under consideration the equivalent strain rate is

$$
\xi_{e q}=\sqrt{\frac{2}{3}\left(\xi_{\zeta}^{2}+\xi_{\eta}^{2}\right)}=\frac{|\zeta+\mathrm{d} s / \mathrm{d} a|}{\sqrt{3}(\zeta a+s)} \frac{\mathrm{d} a}{\mathrm{~d} t} .
$$

Here Eq. (7) has been taken into account.

It is assumed that the material obeys the double shearing model [15]. In particular, the yield criterion in the plane polar coordinate system reads

$$
\left|\sigma_{r}-\sigma_{\theta}\right|+\left(\sigma_{r}+\sigma_{\theta}\right) \sin \varphi=2 k \cos \varphi .
$$

In the case of the original double shearing model proposed for granular materials, $k$ and $\varphi$ are material constants. In the case of metals, it is natural to assume that $k$ depends of the equivalent strain, $\varepsilon_{e q}$ [13]. The equivalent strain is defined by the following equation

$$
\frac{d \varepsilon_{e q}}{d t}=\xi_{e q}
$$

The equations that connect stresses and strain rates are [15]

$$
\begin{aligned}
& \xi_{r r}+\xi_{\theta \theta}=0, \\
& \xi_{r \theta} \cos 2 \psi-\frac{1}{2}\left(\xi_{r r}-\xi_{\theta \theta}\right) \sin 2 \psi+ \\
& \sin \varphi\left(\omega_{r \theta}+\frac{d \psi}{d t}\right)=0 .
\end{aligned}
$$


Here $\xi_{r \theta}$ and $\omega_{r \theta}$ is the shear strain rate and material spin in the plane polar coordinate system, respectively, $\psi$ is the angle which the axis corresponding to the major principal stress makes with the $r$-direction, and $d / d t$ denotes the convected derivative. The constitutive equations should be supplemented by the equations of equilibrium. It will be seen later that there is one nontrivial equation in the form

$$
\frac{\partial \sigma_{r}}{\partial r}+\frac{\sigma_{r}-\sigma_{\theta}}{r}=0
$$

\section{General solution}

The first equation in Eq. (11) is the equation of incompressibility. This equation is satisfied by the mapping given by Eq. (2). Consider the second equation in Eq. (11). It follows from Eq. (2) that coordinate curves of the $(r, \theta)$ coordinate system are trajectories of the principal strain rates. Therefore, $\xi_{r \theta}=0$. Since these coordinate curves are also trajectories of the principal stresses, $\psi=0$ or $\psi=\pi / 2$. In either case $\sin 2 \psi=0$ and $d \psi / d t=0$. Finally, $\omega_{r \theta}=0$ since coordinate curves of the $(r, \theta)$ coordinate system coincide with coordinate curves of the Lagrangian coordinate system. Thus the second equation of Eq. (11) is satisfied. The equivalent strain rate in Eq. (10) can be eliminated by means of Eq. (8). Then, in domains where $\xi_{r}$ (and $\xi_{\theta}$ ) does not change its sense, Eq. (10) can be integrated using the initial condition $\varepsilon_{e q}=0$ at $a=0$ and Eq. (3) to give

$$
\varepsilon_{e q}=\frac{1}{\sqrt{3}}|\ln [4(\zeta a+s)]|
$$

Replacing in Eq. (12) differentiation with respect to $r$ with differentiation with respect to $\zeta$ by means of Eq. (4) yields

$$
\frac{\partial \sigma_{r}}{\partial \zeta}+\frac{a\left(\sigma_{r}-\sigma_{\theta}\right)}{2(\zeta a+s)}=0
$$

In order to find the stress solution, it is necessary to consider the cases $\sigma_{r}-\sigma_{\theta}>0$ and $\sigma_{r}-\sigma_{\theta}<0$ separately. Assume that $\sigma_{r}-\sigma_{\theta}<0$. Then, solving Eq. (9) for $\sigma_{\theta}$ results in

$$
\sigma_{\theta}=\frac{(1-\sin \varphi)}{(1+\sin \varphi)} \sigma_{r}+\frac{2 k \cos \varphi}{(1+\sin \varphi)}
$$

The inequality $\sigma_{r}-\sigma_{\theta}<0$ corresponds to $\ln [4(\zeta a+s)]>0$. Therefore, eliminating in Eq. (14) the stress $\sigma_{\theta}$ by means of Eq. (15) and replacing differentiation with respect to $\zeta$ with differentiation with respect to $\varepsilon_{e q}$ by means of Eq. (13) leads to

$$
\frac{\partial \sigma_{r}}{\partial \varepsilon_{e q}}=\frac{\sqrt{3} k \cos \varphi}{(1+\sin \varphi)}-\frac{\sqrt{3} \sin \varphi}{(1+\sin \varphi)} \sigma_{r}
$$

Since $k$ is a given function of $\varepsilon_{e q}$ this equation can be immediately integrated.

The case $\sigma_{r}-\sigma_{\theta}>0$ can be treated in a similar manner. As a result,

$$
\frac{\partial \sigma_{r}}{\partial \varepsilon_{e q}}=-\frac{\sqrt{3} k \cos \varphi}{(1-\sin \varphi)}+\frac{\sqrt{3} \sin \varphi}{(1-\sin \varphi)} \sigma_{r} .
$$

\section{Ideal flow solution for bending under tension}

The ideal flow conditions require that the neutral line coincides with a material line. It is seen from Eq. (8) that the neutral line is determined by the equation $d s / d a=-\zeta$. Since $\zeta$ is a Lagrangian coordinate, the ideal flow requirement is $d s / d a=-\zeta_{0}$ where $\zeta_{0}$ is constant. Then, integrating with the use of Eq. (3) gives

$$
s=\frac{1}{4}-a \zeta_{0} .
$$

It is evident that $\sigma_{r}-\sigma_{\theta}<0$ in the range $0 \geq \zeta \geq \zeta_{0}$. Therefore, Eq. (16) is valid in this range. The boundary condition to this equation is $\sigma_{r}=0$ for $\zeta=0$. Then, solving Eq. (16) with the use of this boundary condition gives

$$
\begin{aligned}
& \sigma_{r}=\frac{\sqrt{3} \cos \varphi}{(1+\sin \varphi)} \exp \left[-\frac{\sqrt{3} \sin \varphi}{(1+\sin \varphi)} \varepsilon_{e q}\right] \times \\
& \int_{\varepsilon_{1}}^{\varepsilon_{e q}} k(z) \exp \left[\frac{\sqrt{3} \sin \varphi}{(1+\sin \varphi)} z\right] d z .
\end{aligned}
$$

Here $\varepsilon_{1}$ is the value of $\varepsilon_{e q}$ at $\zeta=0$ (or $r=r_{A B}$ ) and $\mathrm{z}$ is a dummy variable of integration. In the case under consideration, $\varepsilon_{e q}=0$ at the neutral line. Therefore, it follows from Eq. (19) that

$$
\sigma_{n}=\frac{\sqrt{3} \cos \varphi}{(1+\sin \varphi)} \int_{\varepsilon_{1}}^{0} k(z) \exp \left[\frac{\sqrt{3} \sin \varphi}{(1+\sin \varphi)} z\right] d z
$$

where $\sigma_{n}$ is the value of $\sigma_{r}$ at $\zeta=\zeta_{0}$. Equation (17) is valid in the range $\zeta_{0} \geq \zeta \geq-1$. The boundary condition to this equation is $\sigma_{r}=\sigma_{n}$ for $\zeta=\zeta_{0}\left(\right.$ or $\left.\varepsilon_{e q}=0\right)$. Then, solving Eq. (17) with the use of this boundary condition gives 


$$
\begin{aligned}
& \sigma_{r}=\exp \left[\frac{\sqrt{3} \sin \varphi}{(1-\sin \varphi)} \varepsilon_{e q}\right] \times \\
& \left\{\sigma_{n}-\frac{\sqrt{3} \cos \varphi}{(1-\sin \varphi)} \int_{0}^{\varepsilon_{e q}} k(z) \exp \left[-\frac{\sqrt{3} \sin \varphi}{(1-\sin \varphi)} z\right] d z\right\} .
\end{aligned}
$$

Using this equation it is possible to find $p$ as

$$
\begin{aligned}
& p=-\exp \left[\frac{\sqrt{3} \sin \varphi}{(1-\sin \varphi)} \varepsilon_{2}\right] \times \\
& \left\{\sigma_{n}-\frac{\sqrt{3} \cos \varphi}{(1-\sin \varphi)} \int_{0}^{\varepsilon_{2}} k(z) \exp \left[-\frac{\sqrt{3} \sin \varphi}{(1-\sin \varphi)} z\right] d z\right\} .
\end{aligned}
$$

Here $\varepsilon_{2}$ is the value of $\varepsilon_{e q}$ at $\zeta=-1$ (or $r=r_{C D}$ ). In Eqs. (21) and (22) it is necessary to eliminate $\sigma_{n}$ by means of Eq. (20). Using Eqs. (5) and (22) it is possible to find $\mathrm{F}$ from Eq. (1). The distribution of the circumferntial stress can be determined from Eqs. (9), (19) and (21). Then, the bending moment is calculated by means of Eq. (6).

The final shape is completely determined by the values of $r_{C D}$ and $\theta_{0}$ at the end of the process. Assume that these values are equal to $r_{f}$ and $\theta_{f}$, respectively, and $a_{f}$ is the value of $\mathrm{a}$ at the end of the process. Then, it follows from Eqs. (5) and (18) that

$$
a_{f}=\frac{H \theta_{f}}{2 L}, \quad \zeta_{0}=\frac{L}{2 H \theta_{f}}\left(1-\frac{r_{f}^{2} \theta_{f}^{2}}{4 L^{2}}\right)-1
$$

\section{Summary}

A new ideal flow solution has been found. Its distinguished feature is that the plasticity model adopted is pressure-dependent. The solution is for the process of bending under tension. The ideal flow paths in this process are obtained if the neutral line is a material line. It has been shown that the position of the neutral line can be found if the initial and final shapes are prescribed (see Eq. (23)). Then, the distribution of the radial stress is given by Eqs, (19) and (21). This distribution depends on two variables, $a$ and $\zeta$. In order to find the dependence of the radial stress on physical quantities, it is necessary to use Eqs. (4), (5) and (18). Once the distribution of the radial stress has been determined, the distribution of the circumferential stress follows from Eq. (9). Then, the bending moment and tensile forces are found from Eqs. (1), (5), (6) and (22).

\section{Acknowledgment}

The research described was supported by the grants RSCF-16-49-02026 (Russia) and INT/RUS/RFBR/P-214 (India).

\section{References}

1. R. Hill, J. Mech. Phys. Solids 15, 223 (1967)

2. O. Richmond, S. Alexandrov, Acta Mech. 158, 33 (2002)

3. S. Alexandrov, Y. Mustafa, E. Lyamina, Meccanica 51, 2235 (2016)

4. I.F. Collins, S.A. Meguid, Trans. ASME J. Appl. Mech. 44, 271 (1977)

5. K. Chung, S. Alexandrov, Appl. Mech. Rev. 60, 316 (2007)

6. O. Richmond, H.L. Morrison, J. Mech. Phys. Solids 15, 195 (1967)

7. H.F. Weinberber, Meccanica 38, 547 (2003)

8. C.J. Cawthorn, E.G. Loukaides, J.M. Allwood, Prog. Eng. 81, 2451 (2014)

9. E. Orowan, Proc. Inst. Mech. Eng. 150, 140 (1943)

10. G.-L. Zhang, Z-W. Wang, S.-H. Zhang, M. Cheng, H.-W. Song, Proc. IMechE, Part B: J. Eng. Manufact. 227, 1023 (2013)

11. W.A. Spitzig, R.J. Sober, O. Richmond, Metallurg. Trans. 7A, 1703 (1976)

12. A.S. Kao, H.A. Kuhn, W.A. Spitzig, O. Richmond, Trans. ASME J. Eng. Mater. Technol. 112, 26 (1990)

13. C.D. Wilson, Trans. ASME J. Appl. Mech. 69, 63 (2002)

14. P.S. Liu, Mater. Sci. Eng. 422A, 176 (2006)

15. A.J.M. Spencer, J. Mech. Phys. Solids 12, 337 (1964)

16. M. Yoshida, F. Yoshida, H. Konishi, K. Fukumoto, Int. J. Mech. Sci. 47, 1885 (2005)

17. M.H. Parsa, S.N.A. Ahkami, Int. J. Mater. Form. Suppl. 1, 173 (2008)

18. M.A. Guler, F. Ozer, M. Yenice, M. Kaya, Steel Res. Int. 81, 801 (2010)

19. S. Alexandrov, K. Manabe, T. Furushima, Arch. Appl. Mech. 81, 1935 (2013)

20. S.A. Kagzi, A.H. Gandhi, H.K. Dave, H.K. Raval, Mech. Adv. Mater. Struct. 23, 80 (2015)

21. S. Alexandrov, J.-H. Kim, K. Chung, T.-J. Kang, J. Strain Anal. Eng. Des. 41, 397 (2006) 